\title{
Reconstruction of Wavelet Coefficients
}

\author{
K. Mathew \\ Karpagam University, Coimbatore, Tamilnadu- \\ 641021, India
}

\author{
S. Shibu, Ph.D \\ K.R.Gouri Amma College Of Engineering For \\ Women, Cherthala, Kerala, India
}

\begin{abstract}
A comparative study of different methods of reconstruction of wavelet coefficients is presented. The following are the different techniques for the reconstruction of wavelet coefficients. To start with, we show how to design and construct Daubechies four coefficient wavelet system which are orthogonal and compactly supported wavelets. Then we outline the multi resolution analysis of wavelets using Mallat transform. Multi resolution analysis can be illustrated by the decomposition and reconstruction of wavelet system using Laplacian Pyramid. To construct wavelet systems with finite support and regularity using orthonormal and interpolariting units, only multicomponent wavelets are possible. When image function is expressed in terms of scaling functions and wavelet functions of higher resolution, we need to consider only few wavelet coefficients and wavelet coefficients are dominant only near edges. The wavelet coefficient near edges can be estimated using wavelet transform maxima and statistical inference of coefficients using Markov tree model. An alternate method is the reconstruction of wavelet coefficients using total variation minimisation. If we use thresholding, so that when we neglect wavelet coefficients having values less than a threshold value there is ringing across edges. Hence we propose an improved algorithm of reconstruction of wavelet coefficients using zero padding and cycle spinning. PSNR of images with wavelet based interpolation and denoising by cycle spinning is moderately high.
\end{abstract}

\section{Keywords}

Wavelets of finite support, multiresolution analysis, multicomponent wavelets, cycle spinning, Markov tree model, wavelet transform maxima, total variation minimisation.

\section{INTRODUCTION}

Wavelets are a powerful tool for digital image analysis or time frequency analysis of image. A wavelet is a small wave, which is oscillatory and it contains both the analysing shape and the window (a finite support). One can construct wavelets $\psi$ such that the dilated and translated family $\left\{\psi_{j, n}(t)=\frac{1}{\sqrt{2^{j}}} \psi\left(\frac{t}{2^{j}}-n\right)\right\}_{j, n \in z^{2}}$ is an orthonormal basis of $\mathrm{L}^{2}(\mathrm{R})$. Orthogonal wavelets dilated by $2^{j}$ carry signal variation at resolution $2^{-j}$.The construction of these bases is related to multiresolution signal approximation.n-refers to the translation of signal and $\mathrm{j}$ refers to the scale and $\mathrm{n}$ refers to time location. The wavelet function $\psi_{j, n}(t)$ has a time spread $\sigma_{+}\left(j_{n}\right)$ related as $\sigma_{t}{ }^{2}(j, n)=\int t^{2}|\psi(t)|^{2} d t$ and energy (frequency)spread $\sigma_{w}\left(\hat{\psi_{j, n}}\right)$ is given by

$$
\sigma_{\omega}^{2}\left(\hat{\psi}_{j, n}^{\wedge}\right)=\frac{1}{2 \pi} \int\left(\omega-\omega_{0}\right)\left|\hat{\varphi_{(\omega)}}\right|^{2} d \omega 2^{j} \quad \text { is the }
$$

dilation parameter which changes the support of $\psi$ in time and rescales $\psi$ and changing the translation parameter $\mathrm{n}$ makes $\psi_{j, n}$ changes its location. It is observed that small scales corresponds to high frequency. Wavelet functions are located both in time and frequency, but it cannot be exact localisation due to uncertainty principle so instead of exact localisation, the function is restricted to wavelet Heisenberg box. The localisation measures $\sigma_{x}\left(\psi_{j n}\right)$ and $\sigma_{\omega}\left(\hat{\psi}_{j n}\right)$ are often represented by rectangles in time frequency plane. These rectangles have same, but their sides are stretched and contracted by the factor $2^{j}$ and $\frac{1}{2^{j}}$. All information about the transformed signal is preserved when the wavelet transform is sampled on certain discrete subset of time frequency plane. The values of continuous transform in those points are coefficients of a corresponding wavelet basis series expansion. The image function is decomposed in terms of orthonormal basis function

$$
\begin{aligned}
& f=\sum_{j, n} C_{j, n} \psi_{j, n} \text { where } \\
& C_{j, n}=\left\langle f, \psi_{j n}\right\rangle=\int \psi_{j n}{ }^{*}(t) f(t) d t
\end{aligned}
$$

This is a doubly infinite sum over both time index $\mathrm{n}$ and scale index $\mathrm{j}$ and however sum can be made finite with no error because in expansion, only few coefficients are dominant. Multiresolution analysis allows us to decompose a signal or image function into approximation and details. These coefficients can be computed using various bank filters or Daubechies filters or Laplacian pyramid structure. Consider one dimensional image function

$$
f(t)=\sum_{k} C_{j_{0}, k} \phi_{j_{0}, k}+\sum_{j_{0} \leq j<J} \sum_{k} d_{j k} \psi_{j k}
$$

where $\phi$ called scaling function and $\psi$ is is called wavelet. $\phi_{j_{0}, k}$ and $\psi_{j k}$ are generated by translation and dilations of $\psi$. The expansion functions which are composed of integer translations and binary scaling of the real square integral

functions, then $\phi_{j_{0}, k}=2^{\frac{j_{0}}{2}} \phi\left(2^{j_{0}} x-k\right) \quad$ where $j_{0}, k \in Z \quad \phi(n) \in L^{2} \quad j_{0}$ represent width of function $\phi_{j_{0}, k}, 2^{\frac{j_{0}}{2}}$ control amplitude of the function and shape of 
function changes withjo. $\psi_{j, k}=2^{\frac{j}{2}} \psi\left(2^{j} x-k\right)$, $C_{j_{0}, k}=\int \phi_{j_{0, k}}^{*} \psi d t$ and $d_{j, k}=\int \phi_{j, k}^{*} \psi d t$. The scaling functions and wavelet functions obeying the following rules:

1) Rested properly $v_{j} \subset v_{j+1}$ for each $j \in Z$ where $v_{j}$ the subspace spanned by scaling functions corresponding to resolution $\mathrm{j}$.

2) If $\mathrm{f}(\mathrm{t}) \in V_{j}$ if and only if $f(2 t) \in V_{j+1}$ for all $j \in Z$

3) $\bigcap_{j \in Z} V_{j}=\{0\}$ and $\underset{j \in Z}{\cup} V_{j}=L^{2}$

4) There exists a function $\theta$ such that $\{\theta(t-k)\}_{k \in Z}$ is a Riesz basis of $V_{0}$. The nesting property $V_{J} \subset V_{J+1}$ implies that the approximation at a resolution $2^{-j}$ contain all necessary informationto compute a finer resolution $2^{-j-1}$. Dilating function in $V_{j}$ by 2 enlarges the detail by 2 .

\section{CONSTRUCTION OF WAVELET SYSTEMS-THE DAUBECHIES FOUR COEFFICIENT WAVELET SYSTEM}

The scaling function $\phi(t)$ is expanded as $\phi(t)=\sum_{k=-\infty}^{\infty} C_{j_{0}, k} \phi\left(2^{j} t-x\right)$.The constant coefficients $C_{j_{0}, k}$ are derived by imposing certain conditions on the scaling function. One such condition is orthonormal property on scaling function and its translates.

i.e. $\quad \int_{-\infty}^{\infty} \phi(t) \phi(t+l) d t=\delta_{o, l} \quad ; \quad l \in Z$

The wavelet $\psi(x)$ is orthogonal to scaling function and is fixed by $\psi(t)=\sum_{k=-\infty}^{\infty} d_{j k} \psi\left(2^{j} t-k\right)$

$\langle\phi(t), \psi(t)\rangle=\int \psi^{*}(t) \phi(t) d t=0$.The $\quad$ coefficient $C_{j_{0}, k}$ and $d_{j, k}$ form a pair of quadrature mirrors. In order to uniquely define all scaling functions of given shape, the area under the scaling function is normalized to unity i.e. $\int_{-\infty}^{\infty} \phi(t) d t=1$ and the correspondingcondition on expansion coefficient is of the form

$$
\sum_{k=-\infty}^{\infty} C_{j_{0}, k}=2
$$

If denote $C_{j_{0}, k}$ as $a_{k}$, this equation becomes $\sum a_{k}=2$ for the scaling function to be orthogonal to its integer translates, i.e. $\int_{-\infty}^{\infty} \phi(t) \phi(t+l) d t=\delta_{o, l}$.

On the expansion coefficients, this yields the condition

$\sum C_{j_{0}, k} C_{j_{0}, k+2 l}=2 \delta_{o, l}$

and $\sum a_{k} a_{k+2 l}=2 \delta_{0, l}$. These conditions are not sufficient

to solve the coefficients .For that we require that scaling function is exactly represented by polynomial of order up to $p$ i.e.

$f(t)=\alpha_{0}+\alpha_{1} t+\alpha_{2} t^{2}+\ldots \ldots \ldots \ldots \ldots \ldots . . .+\alpha_{p-1} t^{p-1}$

and

$f(t)=\sum_{k=-\infty}^{\infty} C_{j_{0}, k} \phi\left(2^{j_{0}} t-k\right) \quad$ especially when $j_{0}=1$, denote $C_{j_{0}, k}=a_{k}$

$f(t)=\sum_{k=-\infty}^{\infty} a_{k} \phi(t-k)$.Since $\int f(t) d t=0$, from the polynomial form of the scaling function we get

$\int_{-\infty}^{\infty} \psi(t) t^{l} d t=0$ where $1=0,1,2, \ldots \ldots, \mathrm{p}-1$ i.e. $\mathrm{p}$ moments of wavelet must be zero and in terms of coefficient we have

$$
\sum_{k=-\infty}^{\infty}(-1)^{k} a_{k} t^{l}=0 \text { for } \mathrm{l}=0,1,2, \ldots \ldots ., \mathrm{p}-1
$$

We consider the construction of scalar function coefficients. To solve the equations (1),(2) and (3) and it is easiest to include $1=0$ in eq (3) and exclude the nonlinear eq (2), we get

$$
\begin{aligned}
& a_{0}+a_{1}+a_{2}+a_{3}=2 \\
& a_{0}^{2}+a_{1}^{2}+a_{2}^{2}+a_{3}^{2}=2 \\
& a_{0}-a_{1}+a_{2}-a_{3}=0 \\
& -a_{1}+2 a_{2}-3 a_{3}=0
\end{aligned}
$$

Solving these equations, we get

$$
\begin{aligned}
& a_{0}=a_{1}-\frac{1}{2}, \quad a_{2}=\frac{3}{2}-a_{1}, \quad a_{3}=1-a_{1} \quad \text { and } \\
& a_{1}=\frac{3 \pm \sqrt{3}}{4} \quad a_{0}=\frac{1 \pm \sqrt{3}}{4} \\
& a_{2}=\frac{3 \mp \sqrt{3}}{4} \quad \text { Thus } \quad a_{3}=\frac{1 \mp \sqrt{3}}{4} .
\end{aligned}
$$

One set of equation is antithesis to the other. If one leads to scaling function $\phi(x)$, other leads to $\phi(-x)$. Both satisfies conditions of normality and orthonormality and representation of linear equation. If we choose $a_{0}=\frac{1+\sqrt{3}}{4}$, 
$a_{1}=\frac{3+\sqrt{3}}{4}, a_{2}=\frac{3-\sqrt{3}}{4}, a_{3}=\frac{1-\sqrt{3}}{4}$, the scalar function at integer points are

$\left[\begin{array}{cccc}a_{0}-1 & 0 & 0 & 0 \\ a_{2} & a_{1}-1 & a_{0} & 0 \\ 0 & a_{3} & a_{2}-1 & a_{1} \\ 0 & 0 & 0 & a_{3}-1\end{array}\right]\left[\begin{array}{c}\phi(0) \\ \phi(1) \\ \phi(2) \\ \phi(3)\end{array}\right]=$

$\left[\begin{array}{l}0 \\ 0 \\ 0 \\ 0\end{array}\right]$

From which $\quad \phi(0)=0, \quad \phi(3)=0_{\text {and }}$ $\phi(2)=\frac{1-\sqrt{3}}{1+\sqrt{3}} \phi(1)$ and $\phi(1)=\frac{1+\sqrt{3}}{2}$ and hence $\phi(2)=\frac{1-\sqrt{3}}{2}$ and hence $\phi(x)$ at half integer $\phi\left(\frac{1}{2}\right)=\frac{2+\sqrt{3}}{4}, \phi\left(\frac{3}{2}\right)=0, \quad \phi\left(\frac{5}{2}\right)=\frac{4-\sqrt{3}}{4}$ and all other half integer values are zero and it is found that the scaling function is zero outside the interval $[0,3]$ i.e. it has compact support.

\section{ESTIMATING WAVELET COEFFICIENTS BASED ON LAPLACIAN PYRAMID}

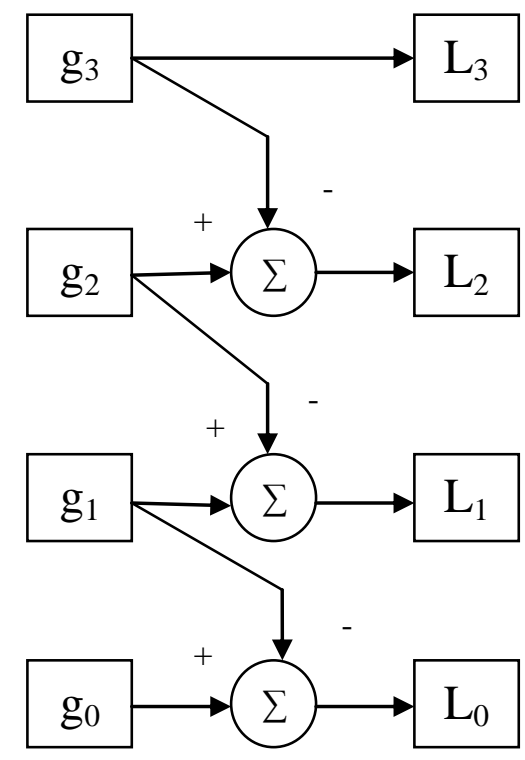

Three level decomposition of images

that neighbouring pixels are highly correlated. So to design an efficient compressed code we use a representation which in effect, decorrelate the image pixel. This is achieved through predictive and through image pixel. The process of image decomposition and reconstruction based Laplacian pyramid is shown in the figure 1 .

In this figure $g_{0}$ is an image and $g_{1}$ is the resulting image applying an appropriate Low pass filter and down sampling $g_{0}$. Prediction error $L_{0}=g_{0}$-upsampling $g_{1}, g_{1}$ is itself low pass filtered and down sampled to obtain $g_{2}$. $L_{1}=g_{1}-$ upsampling $g_{2}$. By repeating these steps we get $\quad L_{2}, L_{3}, \ldots \ldots \ldots \ldots \ldots . . . L_{K+1}$ where $L_{k+1}=g_{k}$-upsampling $g_{k+1}$. So we get a sequence of two dimensional arrays $g_{0}, g_{1}, \ldots \ldots \ldots g_{n}$ and prediction errors $L_{0}, L_{1}, \ldots \ldots . L_{k+1}$.In the above

implementation each $\lambda$ smaller than its predecessor by a factor $\frac{1}{2}$. This image representation called Laplacian pyramid. Those low pass filtered images $g_{0}, g_{1}, \ldots \ldots \ldots g_{n}$ form a Gaussian Pyramid.

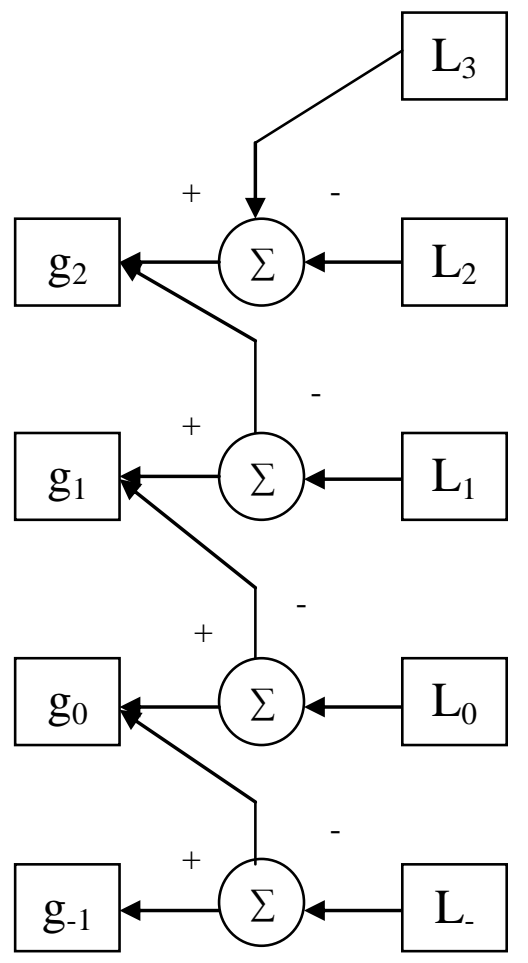

The reconstruction of images

For the reconstruction of decomposed image,we start with

To reconstruct the digital images, we per and reconstruction of digitized image by Pyramid algorithms. A common characteristic of images is to image $g_{n+1}$ to serve as prediction image 
for $g_{n}$,we choose $L_{n}=g_{n}$. So we upsample $\mathrm{L}_{\mathrm{n}}$ and it to $\mathrm{L}_{\mathrm{n}-1}$. Then upsample this and add to $\mathrm{L}_{\mathrm{n}-2}$ and so on. Until the level 0 is reached and $g_{0}$ is recovered. But our aim is to obtain higher resolution image than $g_{0}$.Predictive high resolution image

$$
g_{-1}=L_{-1}+\text { upsampling } g_{0}
$$

\section{MULTI COMPONENT WAVELET}

Wavelet theory is based on the multi resolution analysis. Multiresolution analysis is generated by one scaling function and dilates and translates of only one wavelet $\psi \in L^{2}(R)$ form a stable basis of $\mathrm{L}^{2}(\mathrm{R})$. If we require short support, orthonormal MRA, regular and interpolating basic function, above scaling functions and wavelet function formed from a single wavelet is not possible. But if we use multicomponent wavelet, it is possible to construct scaling functions and wavelet functions having the properties of regularity,orthonormality, and interpolating nature. Multi wavelets are constructed by means of a scaling vectors and these scaling vectors satisfy the equations of the form

$\phi(t)=\sum_{k \in Z} A_{K} \phi(2 x-t)$.Almost all important properties

of the scaling vector carry over to the resulting multiwavelets, $A_{k}, k \in Z$ is a real $r x r$ matrix,the mask $\bar{A}=\left(A_{K}\right), k \in Z$ and length of $\bar{A}$ is defined as $l(\bar{A})=\min \left\{|R-L|:\left\{k: A_{k} \neq 0\right\} \subset[L, R]\right\} . \quad$ The fourier transform yield $\hat{\phi(\omega)}=\frac{1}{2} \bar{A} e^{\frac{-i \omega}{2}} \hat{\phi}\left(\frac{\omega}{2}\right)$ with the equation $\overrightarrow{A(z)}=\sum_{k \in Z} A_{k} z^{k} ; z \in T$, T denotes unit circle. In general, r-scaling vectors for the basis of r-multi wavelets, ie vectors $\bar{\psi}=\left\{\psi_{0}, \psi_{1} \ldots \ldots \ldots \ldots \ldots . . . \psi_{r-1}\right\}^{T}$ of $L_{2}(R) \quad$ functions for which $\left\{\psi_{0}\left(2^{j},-k\right), \ldots \ldots \ldots \psi_{r-1}\left(2^{j},-k\right) \mid j, k \in Z\right\}$ forms a Riesz basis of $L_{2}(R) \cdot \psi$ can be represented as $\psi \overline{(x)}=\sum_{k \in Z} B_{k} \phi(2 x-k)$ with $r x r$ real matrices $\mathrm{B}_{\mathrm{k}}$. By applying fourier transform to this equation $\hat{\psi}(\omega)=\frac{1}{2} \bar{B} e^{-i \frac{\omega}{2}} \hat{\phi}\left(\frac{\omega}{2}\right) \quad$ with $\quad$ the equation $\bar{B}(z)=\sum_{k \in Z} B_{k} z^{k}$. Thus it is possible to construct regular, orthonormal and interpolating wavelets which have finite support if we use multicomponent wavelets.

\section{DETERMINATION OF WAVELET COEFFICIENT AND RECONSTRUCTION OF WAVELETS USING HIDDEN MARKOV TREE MODEL}

In statistical image processing, we can have an image $\mathrm{x}$ as a realization of a random field with joint probability density function $f(x)$. We can model local joint statistics of image pixels in the spatial domain as Markov random field model. In wavelet transform, wavelet coefficient measures the differences at different scales of resolution. The portions of image which do not vary significantly from scale to scale (smooth region) will be expressed by few large values at coarse scales. The portions of image that do vary from scale to scale are regions around edges and are represented by large values at each scale in the wavelet transform.

We demand that the following primary properties are obeyed by wavelet transform.

(1) Locality: Each wavelet coefficient represents image content local in space and frequency.

(2) Multiresolution. The wavelet transform represents the image at nested set of scales.

(3) Edge detection : Wavelets act as a local edge detectors. The edges in image are represented by large wavelet coefficients at corresponding location.

(4) The wavelet coefficient will have only few significant coefficients at smooth regions and wavelet coefficients is large only if edges are present within support of wavelet. (5) The wavelet coefficient tends to be approximately decorrelated. The above primary properties give wavelet coefficients of natural image significant statistical structure and they are listed as the following secondary properties (1) The wavelet coefficients have peaky heavy tailed marginal distribution ,or distribution is non Gaussian. (2) Magnitudes of the wavelet coefficient of real image decay exponentially across scales. The persistence of large/small wavelet coefficient magnitude becomes exponentially stronger at finer scales.

To accommodate the non Gaussian nature of wavelet coefficient, we can model the marginal probability density function of each coefficient as a Gaussian mixture density with a hidden state of a Markov tree. In a Markov process, the transition probability depends on its current state and parent state. On a hidden Markov tree model we do not know explicitly the state of the process. The parent of a particular state is the coefficient of the next coarsest scale density before it. The probability densities were chosen to be Gaussian with means and variance according to the state. The probability density function of each wavelet is selected as a Gaussian mixture density with a hidden binary state that combines two components. One component features large variance and has large non zero coefficients with small weights (only few coefficients) and the other has small variance with small and zero valued coefficients of large weights (large number of coefficients). We associate to each wavelet coefficient $\theta_{\mathrm{n}}$, an unobserved hidden state $S_{n} \in(S, L)$, the value of $S_{n}$ determine which of the two component of the mixture, model is used to generate $S_{n}$ 
i.e. $f\left(\theta_{n} \mid S_{n}=S\right)=N\left(0, \sigma_{s, n}^{2}\right)$

$f\left(\theta_{n} \mid S_{n}=L\right)=N\left(\sigma, \sigma_{l, n}^{2}\right)$

Probability of the states are given by $p\left(S_{n}=S\right)=P_{n}^{S}$ and $p\left(S_{n}=L\right)=P_{n}^{l}$ with the condition $p_{n}^{S}+p_{n}^{L}=1$. Persistence property induces a Markov tree where the state $S_{n}$ of a coefficient $\theta_{\mathrm{n}}$ is affected only by the state $S_{\mathrm{P}(\mathrm{n})}$ of its parent $\mathrm{P}(\mathrm{n})$. Markov model is completely determined by the set of state transition matrices for different coefficient $\theta_{\mathrm{n}}$ at wavelet scale $1 \leq j \leq J$,

$$
A_{n}=\left[\begin{array}{ll}
p_{n}^{S \rightarrow S} & p_{n}^{S \rightarrow L} \\
p_{n}^{L \rightarrow S} & p_{n}^{L \rightarrow L}
\end{array}\right]
$$

The persistence property implies that $p_{n}^{L \rightarrow L}$ and $p_{n}^{S \rightarrow S}$ are significantly larger than their complements. The probability density for any hidden state can be obtained as

$$
p\left(S_{n}=L\right)=P_{p(n)}^{S} p_{n}^{S \rightarrow L}+P_{p(n)}^{L} p_{n}^{L \rightarrow L}
$$

The hidden Markov tree model parameters include the probabilities for the hidden state $\left\{p_{1}^{S}, p_{1}^{l}\right\}$,state transition matrix $\mathrm{A}_{\mathrm{n}}$, the Gaussian distribution variance $\left\{\sigma_{L, n}^{2}, \sigma_{S, n}^{2}\right\}$ for each of wavelet coefficients $\theta_{\mathrm{n}}$. We can make the coefficient dependent parameters, equal for all coefficients within the scale and the magnitude of coefficients decrease exponentially decrease across scales,ie

$$
\begin{aligned}
& \sigma_{L, j}^{2}=C_{\sigma_{L}} 2^{-j \alpha_{L}} \text { and } \\
& \sigma_{S, j}^{2}=C_{\sigma S} 2^{-j \alpha_{S}}
\end{aligned}
$$

Also we can demand weaker persistence in the coarsest scales. Therefore the value of the state transition matrices $A_{j}$ follow a model that strengthen the persistence at finer scale.

This shows that the probability that $S_{n}=L$, given

$S_{P(n)}=L$ is $\frac{1}{2}$ and $p_{j}^{L \rightarrow L}=\frac{1}{2}+C_{L L} 2^{-r_{L} j}$

$p_{j}^{L \rightarrow S}=\frac{1}{2}-C_{L L} 2^{-r_{L} j}, \quad p_{j}^{S \rightarrow S}=1-C_{S S} 2^{-r_{S} j} \quad$ and $p_{j}^{S \rightarrow L}=C_{S S} 2^{-r_{S} j}$

These parameters can be estimated by expectation maximization algorithm. The expectation maximization algorithm is used in the Markov tree model. The algorithm, asserts by finding the set of parameters which would most likely result in set of observed wavelet coefficients. We would take these wavelet coefficients as inputs and produces the state transition probabilities with mean and variance for each coefficient. We can use a training set and EM (Expectation Maximisation) algorithm works by successively iterating model parameters until the minimum error is observed. By this method we produce HL, LH and HH bands of image. Taking the inverse wavelet transform, we get the picture with more than twice the resolution.

Wavelet coefficient in the finest band in our hidden Markov model is done by using the probability distribution relation.

$P\left(\omega_{C}^{k}\right)=\frac{1}{\sigma_{C, m} \sqrt{2 \pi}} e^{\frac{\left(\omega_{C}^{k}-\mu_{j, m}^{k}\right)^{2}}{2 \sigma_{C, m}^{k}}}$ where $\omega_{C}^{k}$ are wavelet coefficients at the finest scale, $\sigma_{C, m}^{k}$ and $\mu_{C, m}^{k}$ are obtained from the training set.

To reconstruct wavelets, we adopt the following steps (i) Create a Hidden Markov tree model of the image statistics of a similar image (training set). This model will consist of transition probability state with certain mean and variance. Training set is tied within the scale. We collect information about all sign changes occurring in the training set wavelet coefficients from parent to child in the Markov tree model (ii) Obtain the wavelet transform of the image to be reconstructed (iii) Iterate the EM algorithm for a single iteration using the Markov tree found during the training (iv) Use the appropriate equation and determine the wavelet coefficient at the finest scale. We can use Gaussian probability distribution to randomly generate a value for wavelet coefficients. Use the probability sign changes and check the coefficients and make the appropriate changes.

\section{RECONSTRUCTION OF WAVELETS USING WAVELET TRANSFORM EXTREMA EXTRAPOLATION AND CYCLE SPINNING}

We propose an algorithm to extract high frequency information so as to sharpen the image by using wavelet transform extrema extrapolation. The wavelet transform modulus maxima has the sharp variations of a signal and their variations characterize the local regularity of the signal. Since edges are points where the image intensity has sharp variation. By means of canny edge detection method, one can detect a two dimensional wavelet transform. The Lipschitz regularity of edge points is derived from the wavelet modulus maxima across scales. The appropriate wavelet can be reconstructed from the wavelet modulus maxima, with no degradation. We can detect points of sharp variation of image $f\left(x_{1}, x_{2}\right)$ by calculating the modulus of its gradient vector

$\bar{\nabla} f=\left(\frac{\partial f}{\partial x_{1}}, \frac{\partial f}{\partial x_{2}}\right)$. The partial derivative of $\mathrm{f}$ in the direction $n$ is calculated as $\frac{\partial f}{\partial n}=\bar{\nabla} f \cdot \bar{n}=\frac{\partial f}{\partial x_{1}} \cos \alpha+\frac{\partial f}{\partial x_{2}} \sin \alpha$.If $\alpha$ is the angle between direction of $\nabla f$ and $\hat{n}$.The absolute value of this $\wedge$

partial derivative is maximum if $n$ is in the same direction 
as $\quad \nabla f$. A multiscalev version of this vedge detector is implemented by smoothing the image function convolution kernel $\theta(\mathrm{x})$ and we can define the wavelets $\psi^{1}$ and $\psi^{2}$ as $\psi^{1}=-\frac{\partial \theta}{\partial x_{1}}$ and $\psi^{2}=-\frac{\partial \theta}{\partial x_{2}}$. The scale varies along the

$$
\begin{aligned}
& \text { binary sequence } \quad\left\{2^{j}\right\}_{j \in Z} \quad \text { and } \\
& \psi_{2^{j}}^{k}\left(x_{1}, x_{2}\right)=\frac{1}{2^{j}} \psi^{k}\left(\frac{x_{1}}{2^{j}}, \frac{x_{2}}{2^{j}}\right) \text { and } \psi_{2^{j}}^{k}=\psi_{2^{j}}^{k}(-x) \\
& \omega^{k} f\left(u, 2^{j}\right)=f * \psi_{2^{j}}^{-}(u)
\end{aligned}
$$

The wavelet transform components are proportional to the coordinate gradient vector of smoothed by $\theta_{2^{j}}^{-}$,

$$
\begin{aligned}
& {\left[\begin{array}{l}
W^{1} f\left(u, 2^{j}\right) \\
W^{2} f\left(u, 2^{j}\right)
\end{array}\right]=2^{j}\left[\begin{array}{l}
\frac{\partial}{\partial u_{1}}\left(f * \theta_{2^{j}}\right)(u) \\
\frac{\partial}{\partial u_{2}}\left(f * \theta_{2^{j}}\right)(u)
\end{array}\right]} \\
& 2^{j} \bar{\nabla}\left(f * \theta_{2^{j}}\right)(u)
\end{aligned}
$$

The modulus of this gradient vector is proportional to wavelet transform modulus

$$
M f\left(u, 2^{j}\right)=\sqrt{\left|w^{\prime} f\left(u, 2^{j}\right)\right|^{2}+\left|w^{2}+\left(u, 2^{j^{\prime}}\right)\right|^{2}}
$$

The angle $\alpha$ of wavelet transform vector is

$$
\alpha(u)=\tan ^{-1}\left(\frac{w^{2} f\left(u, 2^{j}\right)}{w^{1} f\left(u, 2^{j}\right)}\right)
$$

An edge point at the scale $2^{j}$ is a point $\mathrm{u}$ such that $M f\left(u, 2^{j}\right)$ is locally maximum at $\mathrm{u}=\mathrm{v}$ when $u=U+\lambda \bar{n}_{j}(v)$ for $|\lambda|$ is very small enough. These points are called wavelet transform modulus maxima points are distributed along curves that corresponds to the boundary of the angle.

To make the image sharper, we have to effect denoising. The basic principle of denoising is to identify and zero out wavelet wavelet coefficients of the signal which are most likely to contain mostly noise. By preserving significant coefficients, the important high pass feature of the signal, such as discontinuities including edge can be preserved. Denoising by wavelet thresholdings was introduced by Donoho and Johnstone ${ }^{(4)}$. Thresholding in fact introduces ringing effect near the edges. This ringing effect can be minimized by cycle spinning. In cycle spinning, the signal to be denoised is translated by various time shifts. Cycle spinning makes use of the periodic time invariance of the wavelet transform. The wavelet transform is not time invariant and consequently if the noisy signal is shifted in time, denoised and then shifted back. The cycle spinning estimate is

the linear average of the estimates $x^{\wedge}[n]$, $\mathrm{i}=0,1, \ldots \ldots \ldots \ldots . . \mathrm{M}-1$.

Consider an observed signal $y[n]=x[n]+d[n]$ where $d[n]$ is additive noise and $W(x(n))$ is the wavelet transform. Then zeroing out wavelet coefficients, falling below a certain threshold then we take wavelet transform

$\hat{x}[n]=W^{-1}\left(A_{+}[W Y(n)], \mathrm{A}_{+}\right.$is the diagonal thresholding operator that zeroes out wavelet coefficients less than threshold.

In the proposed algorithm, recursive cycle spinning, we generate a sequence of estimates $x_{e}$. The algorithm repeatedly cycles through $\mathrm{M}$ shifts, using the denoised output from one iteration as the input to the next. The algorithm tries to converge to fixed point.

\section{RECONSTRUCTION OF WAVELET USING TOTAL VARIATION MINIMIZATION}

We suggest a revised algorithm to reconstruct wavelet coefficient using a total variation minimization with a view of denoising nearly piecewise smooth function presenting sharp discontinuities. Total variation minimization model was proposed by Rudin, Osher and Fatemi. The image function is decomposed into two components $\mathrm{u}+\mathrm{v}$, where $\mathrm{u}$ is the cartoon part, $\mathrm{v}$ represents the oscillating part- cartoon part contains main features of $\mathrm{f}$ including sharp edges and contours. $\mathrm{u}$ is treated as a function of bound variation $\mathrm{BV}(\Omega)$ or its subset $\operatorname{SBV}(\Omega)$, special functions of bounded variation. If we are given the image function $\mathrm{f} \mathrm{CL}^{2}(\Omega)$ where $\Omega$ is open and bounded domain on $\mathrm{R}^{2}$, for restoration of image or recover the appropriate wavelet showing the main features of the image including edges, $\mathrm{i} t$ is enough to separate is from $\mathrm{f}$, for that we need solve the following minimization problem.

$\underset{u \in B V(\Omega)}{\operatorname{int}} F(u)=\lambda \int|f-u|^{2} d x d y+\int|\nabla u|$

Where $\operatorname{BV}(\Omega)$ is the space of bounded variation, $\lambda$ is a positive constant. $\int_{\Omega}|\nabla u|$ denotes the total variation of $\mathrm{f}$.

The minimiser for $\mathrm{u}$ is satisfying the Euler Lagrange equation associated with the minimizing problem

$u-f=\frac{1}{2 \lambda} \operatorname{div}\left[\frac{\nabla u}{|\nabla u|}\right]$ and the residual function $\mathrm{v}=\mathrm{f}-\mathrm{u}$ can be expressed as $\vec{v}=\operatorname{div} \vec{g}$ where $\vec{g}$ can be represented as $\vec{g}=\frac{1}{2 \lambda} \operatorname{div}\left[\frac{\nabla u}{|\nabla u|}\right], \lambda$ being a constant for u $\in \operatorname{BV}(\Omega)$, its total variation $\int_{\Omega}|\nabla u|$ is finite and can be expressed as

$$
\int|\nabla u|=\stackrel{\sup }{\vec{g}}\left\{\int_{\Omega} u(\operatorname{div} \vec{g}) d x d y ; \vec{g} \in C_{c}^{\prime}\left(\Omega, R^{2}\right)|\vec{g}| \leq 1\right\}
$$




\section{The above total variation follows the condition}

$T V(u)=\int|\nabla u| d x, \quad \Omega$ bounded and convex region of $\mathrm{R}^{2}$ is minimum, subject to the fidelity constraint.

$\|\mathrm{u}-\bar{u}\|_{L}{ }^{2}(\Omega)=\in, \in$ being the estimated error level. The noise is reduced, while the discontinuities including edges are preserved. Although total variation method is relevant in regularising piecewise smooth functions, it will introduce an artifact like staircase effect. Total variation algorithm tends to restore piecewise constant function.

The staircase effect can be minimised by replacing the total variation by the regularized functional $\mathbf{J}_{\beta}$ (u) $=\int_{\Xi} \sqrt{\left((\nabla u)^{2}+\beta^{2}\right)} \mathrm{dx}$, where $\beta$ is a small positive constant. Minimising the new functional the staircase effect can be eliminated. If $\beta$ is very small, the staircase effect is diffused and noise is diffused. If $\beta$ is large, discontinuities are smoothened. The value of $\beta$ is suitably chosen as to eliminate staircase effect, but retaining all the salient features of the image including the edges.

\section{EXPERIMENTAL RESULT AND CONCLUSION}

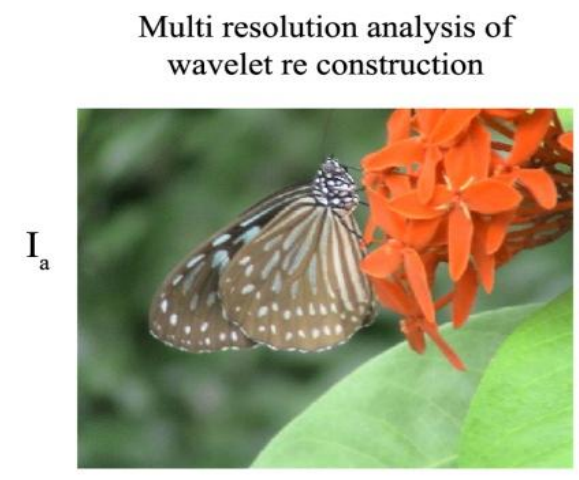

$\mathrm{PSNR}=94.16 \mathrm{~dB}$

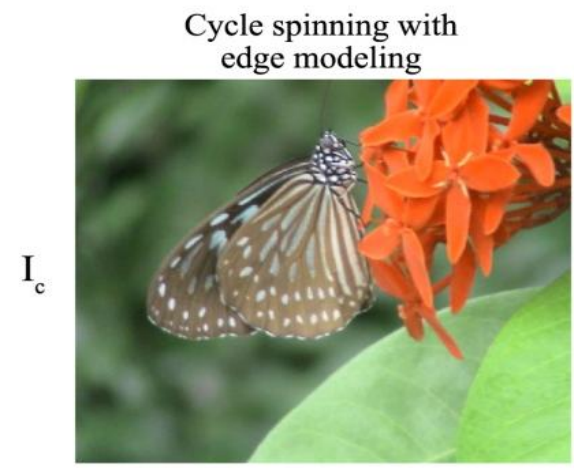

PSNR $=95.518 \mathrm{~dB}$
For digital image analysis, image function is decomposed into scaling functions and wavelet functions, i.e. $\mathrm{f}(\mathrm{t})=\sum_{k} c_{j_{o}, k} \Phi_{j_{o}, k}+\sum_{k} \sum_{j, j_{o}} d_{j, k} \Psi_{j, k} \quad$ where $\quad \mathrm{C}_{j_{o}, k}=$ $\int \Phi_{j_{o}, k}^{*} f(t) d t$ and $\mathrm{d}_{j, k}=\int \Psi_{j, k}^{*} f(t) d t . \mathrm{C}_{j_{o}, k}$ and $\mathrm{d}$ $j, k$ are coefficients of scaling functions and wavelet expansions. For easiness of image analysis, the wavelets should satisfy regularity, orthonormality, and interpolating property in the region of support. We have included different formulations of constructions of wavelets with properties. These wavelet techniques are suitable for resolution enhancement. We suggest revised algorithms for construction of wavelets using hidden Markov tree model, wavelet transform extreme, cycle spinning with edge modelling and total variation minimization denoisity. In fact wavelet coefficient gives high frequency information and as such it will enhance the resolution of images. The results of resolution enhancement using wavelet techniques based on Hidden Markov tree model, wavelet transform extreme cycle spinny total variable minimization are given. It is found that PSNR values of image with these reconstructed wavelets is relatively high.

\section{Wavelet transform extrema}

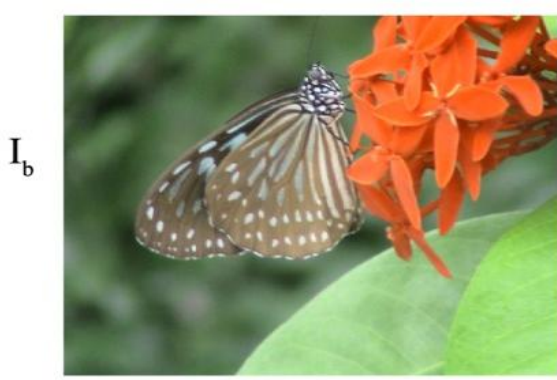

PSNR $=94.72 \mathrm{~dB}$

Total Variation Minimisation

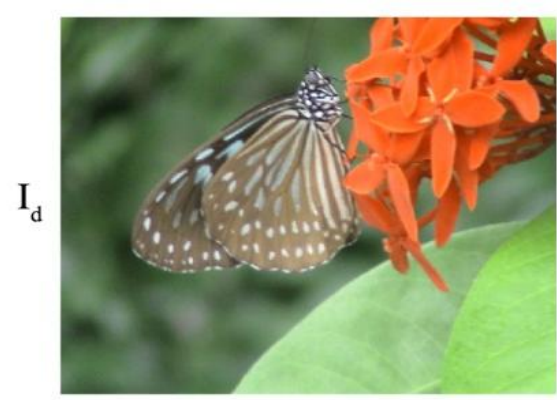

PSNR $=95.21 \mathrm{~dB}$

Fig 2: RECONSTRUCTION OF WAVELET AND RESOLUTION ENHANCEMENT 


\section{REFERENCES}

[1] Mollat S "Multiresolution" approximation and wavelet orthonormal bases of Trans American Society Vol 315(1989) page 68-87.

[2] Mathew K, Dr. Shibu "Wavelet based technique for super resolution images reconstruction "International Journal of Computer application Vol 33(2011) page 11-17.

[3]Mallat S "Wavelet tour of signal processing" Newyork academy 1998

[4]Mallat S "A theory for multiresolution signal decomposition the wavelet representation" IEEE Trans Pattern And Machine Intelligence" Vol 11 pages 674-693 July 1989

[5]I. Daubechies "Ten Lectures on wavelet" Newyork SIAM 1992

[6]Barrus, R.A. Gopinath and H. Guo "Introduction to wavelets and wavelet transform: A primer" Englewood Cliffs NJ Prentice Hall 1998

[7]H.C.Liu , Y.Feng and G.Y. Sun "Wavelet domain image super resolution reconstruction based on Image Pyramid and cycle spinning "Journal of Physics Conference Services 48(2006) pages 417-421 International Symposium of Instrumentation Science and Technologies
[8]J.K.Ronberg, H.Choi and R.G. Bernaut "Bayesian tree structured image modeling using wavelet domain hidden Markov model" IEEE Trans Image Processing 2008 July Pages 1056-1068

[9]Z. Cvetkovie, M. Vetteri "Discrete time wavelet extreme representation" IEEE Trans on signal processing (1995) pages $280-287$

[10] T.N.T Goodman, S.L.LEE "Wavelets of multiplicity" Trans. Of American Math Society-342(1994) Pages 307324

[11]Nhat Nayen and Peyman Milanfar "Wavelet Super resolution circuit system " Signal Process Vol .19 Nov (2000) Pages 321-338

[12]Peter J Burt and Edward H Adelson"Laplacian Pyramid as a compact Image Code" IEEE Transactions on communication Vol.-31 Nov April 1983 Pages 532-540

[13]Luminita A, Vege and Stanley J.Oscher "Image Denoising and decomposition with total variation minimization and oscillatory function"Journal of Mathematical Imaging and Vision 20 Pages 7-18,2004

[14]Sylvian Durand and Jacquies Froment "Reconstruction of wavelet coefficients using total variation minimization " SIAM JSU computer vol 24 No 5 (2003) pages 17541767 